\title{
High expression of HSP47 in ulcerative colitis-associated carcinomas: proteomic approach
}

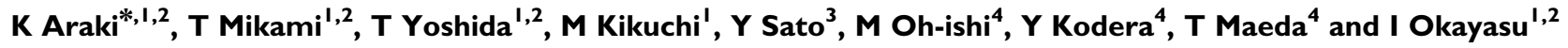 \\ 'Department of Cellular and Histo-pathology, Kitasato University Postgraduate School of Medical Sciences, Sagamihara, Kanagawa, Japan; ${ }^{2}$ Department \\ of Pathology, Kitasato University School of Medicine, Sagamihara, Kanagawa, Japan; ${ }^{3}$ Department of Molecular Diagnostics, Kitasato University School of \\ Allied Health Sciences, Sagamihara, Kanagawa, Japan; ${ }^{4}$ Department of Physics, Laboratory of Biomolecular Dynamics, Kitasato University School of \\ Science, Sagamihara, Kanagawa, Japan
}

BACKGROUND: Ulcerative colitis (UC) is a chronic relapsing inflammatory bowel disease, known to be associated with a markedly increased risk of colorectal carcinoma development.

METHODS: Using proteomic analysis with two-dimensional gel electrophoresis and mass spectrometry, differentially expressed proteins were assessed between UC-associated cancer and sporadic colon cancer cell lines. Western blot and immunostaining were performed for confirming the expression.

RESULTS: Heat-shock protein of $47 \mathrm{kDa}$ (HSP47) was identified as one of the proteins expressed more highly in UC-associated cancer cell lines, and an immunohistochemical examination confirmed significantly higher levels of HSP47 in UC-associated colon cancers than in sporadic counterparts, the expression increasing with a progression of neoplastic lesions. Heat-shock protein of $47 \mathrm{kDa}$ was further found to be coexpressed with type I collagen in the cytoplasm, and both HSP47 and type I collagen were released from cultured cells into the culture medium.

CONCLUSION: These results suggest that overexpression of HSP47 is a unique characteristic of UC-associated carcinoma related to type I collagen synthesis, with possible clinical applications.

British Journal of Cancer (2009) I 0 I, 492-497. doi:I0.1038/sj.bjc.6605 I63 www.bjcancer.com

Published online 14 July 2009

(c) 2009 Cancer Research UK

Keywords: HSP47; ulcerative colitis; adenocarcinoma; collagen; proteomics

Recently, carcinogenesis pathways driven by chronic inflammation have been proposed, with ulcerative colitis (UC)-associated colorectal cancer as one very concrete example (Wong and Harrison, 2001; Okayasu et al, 2002). Ulcerative colitis features chronic, relapsing, and debilitating idiopathic inflammation of the colon mucosa and submucosa, and patients with long-standing UC are at high risk of neoplastic development. Ulcerative colitisassociated carcinomas are often difficult to detect endoscopically and to discriminate from inflammatory regenerative epithelium (Sada et al, 2004; Fujii et al, 2008); hence, a molecular marker is urgently required. Recently, proteomics for large-scale studies of gene expression at the protein level have been viewed as a promising experimental approach to explore the control of biological processes and pathways (Lawrie et al, 2001; Dundas et al, 2005; Kuruma et al, 2005). In this study, using UC-associated cancer cell lines and sporadic colorectal cancer cell lines established by our group (Yamashita et al, 2004), we sought to determine proteins expressed differently between the two cell lines, using proteomics methods. Agarose two-dimensional gel electrophoresis (agarose 2-DE) and liquid chromatography-tandem mass spectrometry (LC-MS/MS) allowed the detection of heat-shock

* Correspondence: K Araki, Department of Pathology, Kitasato University School of Medicine, I-I5-I, Kitasato, Sagamihara, Kanagawa 228-8555, Japan. E-mail: dm07003o@st.kitasato-u.ac.jp

Received 30 March 2009; revised 28 May 2009; accepted 5 June 2009; published online 14 July 2009 protein of $47 \mathrm{kDa}$ (HSP47) as one protein showing an overexpression in UC-associated cancer cell lines. Heat-shock protein of $47 \mathrm{kDa}$ is a molecular chaperone that recognises collagen triple helices in the endoplasmic reticulum (ER) (Nagata et al, 1986; Nakai et al, 1992). Usually, its expression is closely related to that of collagen in collagen-secreting cells, such as fibroblasts. In this study, this expression was further explored in cancer cell lines and immunohistochemically in colon cancer tissues.

\section{MATERIALS AND METHODS}

\section{Cell culture}

Ulcerative colitis-associated cancer cell lines, UCCA-3, UCCA-21, and UCCA-24, and sporadic colorectal cancer cell lines, KE-24, KE-43P, and KE-43W, were earlier established in our laboratory (Yamashita et al, 2004). Cell lines UCCA-3, UCCA-21, UCCA-24, KE-43P, and KE-43W were cultured in Dulbecco's modified Eagle's medium (Gibco, Carlsbad, CA, USA), and cell line KE-24 was cultured in RPMI medium 1640 (Gibco), supplemented with $10 \%$ fetal bovine serum and $1 \%$ penicillin-streptomycin (Gibco), at $37{ }^{\circ} \mathrm{C}$ in an atmosphere of $5 \% \mathrm{CO}_{2}$ and $95 \%$ air in a humidified incubator. When the cells reached the logarithmic growth phase, they were washed with phosphate-buffered saline (PBS), scraped off into a tube, briefly centrifuged, quickly frozen in liquid nitrogen, and stored at $-80^{\circ} \mathrm{C}$ until use. Primary cultures of 
human fibroblasts were kindly provided by Dr Y Kuroyanagi (R\&D Center for Artificial Skin, School of Allied Health Sciences, Kitasato University, Kanagawa, Japan) and similarly cultured in Dulbecco's modified Eagle's medium (Gibco).

\section{Protein extraction}

The cell pellets were sonicated in an extraction solution containing $7 \mathrm{~m}$ urea, $2 \mathrm{~m}$ thiourea, $0.1 \mathrm{~m}$ dithiothreitol, $2.5 \%$ pharmalyte $(\mathrm{pH}$ 3-10), 2\% CHAPS, and Complete Mini EDTA-free (protease inhibitors, Roche Diagnostics, Mannheim, Germany). After centrifugation at 15000 r.p.m. for $20 \mathrm{~min}$, the supernatant was recovered and the protein concentration was measured using a DC Protein Assay kit (Bio-Rad Laboratories, Hercules, CA, USA).

\section{Proteomic analysis}

Proteomic analysis was carried out using agarose 2-DE according to previously published protocols by Oh-Ishi et al (2000) (Tomonaga et al, 2004). The agarose isoelectric focusing gel for the first dimension ( $180 \mathrm{~mm}$ in length and $3.4 \mathrm{~mm}$ in inner diameter) was prepared by stratification of three types of pharmalyte $(\mathrm{pH} 2.5-5$

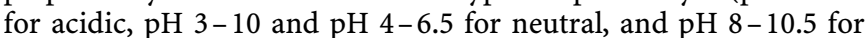
basic solution). The second dimension for SDS-PAGE used $12 \%$ polyacrylamide gels $(200 \times 130 \times 1.3 \mathrm{~mm})$. Cell lysates, equivalent to $500 \mu \mathrm{g}$ of extracted protein, were subjected to all steps of 2-DE, including agarose isoelectric focusing SDS-PAGE, Coomassie Brillant blue staining, and quantitative analysis using Scion Image (Windows version of public domain NIH image program) (Scion Corporation, Frederick, MD, USA). Protein spots with more than two-fold differences in protein intensity were cut from gels and digested with trypsin to produce unique sets of tryptic fragments. The masses of these fragments were determined using the LC-MS/ MS system, consisting of an HPLC system (Nanospace SI-2, Shiseido Fine Chemicals, Tokyo, Japan) and an ion-trap mass spectrometer (LCQ-Deca, Thermo Finnigan, San Jose, CA, USA). A minimum score of 80 was selected as the criterion for the positive identification of proteins. We used the database of the SEQUEST program (an attached software to the LQC-Deca, Thermo Finnigan, San Jose, CA, USA) to identify proteins from measured masses of tryptic peptides and their MS/MS fragments.

\section{Western blot analysis}

Protein samples $(5 \mu \mathrm{g})$ were separated by SDS - PAGEelectrophoresis using $10 \%$ gels, and blotted onto Hybond-P, PVDF membranes (Amersham Pharmacia Biotech, Buckinghamshire, UK). Immunoassays were performed using anti-HSP47 (monoclonal, SPA-470,
1:10 000, Stressgen Biotechnologies, Ann Arbor, MI, USA) and anti- $\beta$-actin (monoclonal, AC-15, 1:10 000, Sigma, St Louis, MO, USA) antibodies. The membranes were then incubated with antimouse immunoglobulin-HRP (DakoCytomation, Glostrup, Denmark), and protein bands were visualised using enhanced chemiluminescence (Amersham Pharmacia Biotech) according to the manufacturer's manual.

\section{Immunocytochemical staining}

The harvested cells were washed and re-suspended in PBS and centrifuged at 1000 r.p.m. for 5 min using Cytospin 2 (Thermo Fisher Scientific Inc., Waltham, MA, USA). Cells on slide glasses were immediately immersed in 95\% ethanol, and immunocytochemical staining was performed using the labelled polymer-peroxidase complex method (EnVision + System HPR; DakoCytomation). Endogenous peroxidase was blocked using $0.3 \%$ hydrogen peroxide for $30 \mathrm{~min}$. After incubation with Protein Block Serum-Free (DakoCytomation), sections were incubated overnight with an anti-HSP47 antibody (monoclonal, SPA-470, $1: 1000$, Stressgen Biotechnologies) at $4{ }^{\circ} \mathrm{C}$. After incubation with a labelled polymer (anti-mouse, EnVision + System HPR; DakoCytomation) for $10 \mathrm{~min}$ at room temperature, 3,3'-diaminobenzidine was used as the chromogen. Nuclei were counter-stained with $0.3 \%$ methylgreen solution.

\section{Immunohistochemical staining}

From the pathology files of the Kitasato University Hospital and the Kitasato University East Hospital, cases of 63 UC-associated lesions and 81 sporadic colon tumour lesions were collected. Given that almost all UC-associated neoplastic lesions were located at the left hemicolon, sporadic colon tumours were mainly collected from cases of the left hemicolon. The clinicopathological data of the cases included in this study are summarised in Table 1. Formalinfixed paraffin-embedded tissue blocks of specimens of $16 \mathrm{UC}$ associated low-grade dysplasias (LGDs), 12 high-grade dysplasias (HGDs), 10 invasive adenocarcinomas, and 25 areas of inflammatory regenerative mucosa, and of 17 cases of sporadic tubular adenoma with LGD, 16 cases of sporadic tubular adenoma with HGD, and 48 cases of sporadic invasive adenocarcinoma were then collected. In addition, 48 normal control mucosa specimens collected at least 5-cm distant from sporadic cancer lesions were also examined. Serial $4-\mu$ m-thick sections were cut for immunohistochemical staining using an EnVision + kit (EnVision + System HPR; DakoCytomation) and anti-HSP47 antibodies at the working dilution (SPA-470, 1:200, Stressgen Biotechnologies).

Table I Clinicopathological data of the patients

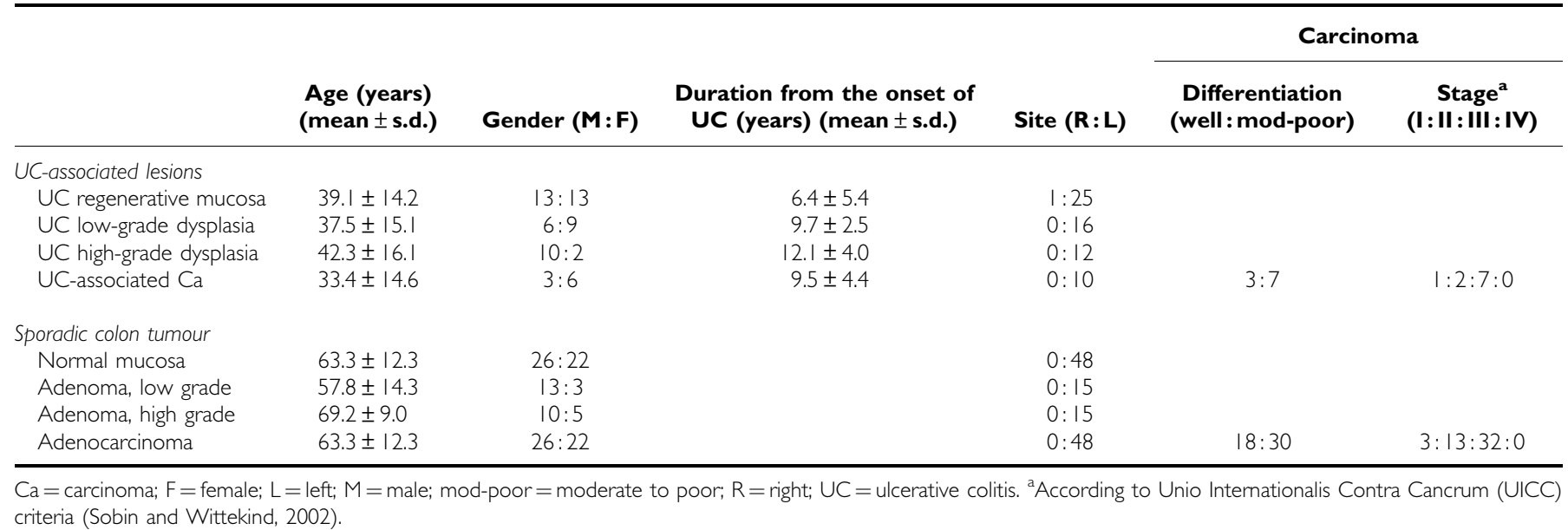


Endogenous peroxidase was blocked by $0.3 \%$ hydrogen peroxide for $30 \mathrm{~min}$. After incubation with Protein Block Serum-Free (DakoCytomation), sections were incubated overnight with antiHSP47 antibody at $4{ }^{\circ} \mathrm{C}$. After incubation with a labelled polymer (anti-mouse, EnVision + System HPR; DakoCytomation) for $30 \mathrm{~min}$ at room temperature, $3,3^{\prime}$-diaminobenzidine was used as the chromogen. Nuclei were counter-stained using Mayer's haematoxylin to facilitate histopathological assessment. Immunoreactivity for HSP47 was evaluated using the scoring system as previously described (Tokuyama et al, 2007), combining staining intensity (none, 0; weak, 1; moderate, 2; intense, 3) with percentages of positive cells $(0,<1 \% ; 1,1-25 \% ; 2,25-50 \% ; 3$, $50-75 \% ; 4,>75 \%)$.

\section{Double immunofluorescence staining of cell lines}

A UC-associated cancer cell line (UCCA-3) and a sporadic carcinoma cell line (KE-24) were cultured on chamber glass slides (Thermo Fisher Scientific Inc.), fixed with 50\% methanol and 50\% acetone for $5 \mathrm{~min}$, and permeabilised in $0.1 \%$ Triton X-100 in PBS for $5 \mathrm{~min}$. The slides were then incubated with anti-monoclonal HSP47 antibody (SPA-470, 1:200, Stressgen Biotechnologies) for $1 \mathrm{~h}$ at room temperature. After washing, anti-collagen type I polyclonal antibodies (COL-1, 1:500, Sigma) were applied for $1 \mathrm{~h}$ at room temperature. The secondary antibodies were rhodaminlabelled anti-mouse IgG and fluorescein isothiocyanate (FITC)labelled anti-rabbit IgG (both 1:500, Molecular Probes, Leiden, The Netherlands), for $30 \mathrm{~min}$ at room temperature. Cell nuclei were counter-stained with DAPI. Negative control staining was performed by omitting the primary antibody incubation. Immediately after treatment, the cell lines were observed under a fluorescence microscope.

\section{ELISA for measuring type I collagen concentrations in culture media}

Cell lines UCCA-3, UCCA-21, UCCA-24, KE-24, KE-43P, and KE$43 \mathrm{~W}$ were cultured in 24-well plates with $1 \mathrm{ml}$ of the culture medium. A human collagen type I ELISA kit (Applied Cell Biotechnologies Inc., Yokohama, Japan) was used to measure the concentration of type I collagen in the culture medium. When the cells reached confluence, $0.2 \mathrm{ml}$ of the culture medium and $0.1 \mathrm{ml}$ of pepsin solution (pepsin $0.6 \mathrm{mg} \mathrm{ml}^{-1}$ dissolved in $150 \mathrm{~mm}$ acetic acid) were mixed, and collagen was digested overnight at $4{ }^{\circ} \mathrm{C}$. Biotinylated anti-type I collagen antibodies were then added and $50 \mu \mathrm{l}$ aliquots of the mixture were applied to microplate wells on which type I collagen was immobilised. After incubation for $1 \mathrm{~h}$ at room temperature and washing, an avidin-horseradish peroxidase conjugate solution was added to the wells, followed by incubation for $1 \mathrm{~h}$ at room temperature. After washing, $50 \mu \mathrm{l}$ of substrate solution was added, and after incubation for $15 \mathrm{~min}$ at room temperature, $50 \mu \mathrm{l}$ of stop solution $\left(1 \mathrm{~N} \mathrm{H}_{2} \mathrm{SO}_{4}\right)$ was added. The concentration of collagen type I was obtained by measuring absorbance at $450 \mathrm{~nm}$ on a microplate reader.

\section{Western blotting of HSP47 in cell culture medium}

Cell lines were cultured in 12-well plates in $2 \mathrm{ml}$ of the culture medium. When they reached confluence, the culture medium was replaced with a serum-free BIO-RICH 1 medium (MP Biomedicals, Irvine, CA, USA) for 4 days. Samples were collected, and $3 \mathrm{ml}$ of extract solution ( $7 \mathrm{M}$ urea, $2 \mathrm{M}$ thiourea, $0.1 \mathrm{M}$ dithiothreitol) was added for overnight incubation at $4{ }^{\circ} \mathrm{C}$. After the solutions were concentrated by acetone precipitation, HSP47 was detected by western blot analysis. As a negative control, the culture medium alone was used.

\section{Statistical analysis}

All statistical tests were conducted using StatView 5.0 software (SAS institute Inc., Cary, NC, USA). Comparisons among more than two groups were assessed using the Kruskal-Wallis test with the Mann - Whitney U-test as a post hoc test. Comparisons between two groups were made using the Mann - Whitney $U$-test. Statistical significance was concluded at $P<0.05$.

\section{RESULTS}

\section{Proteomic analysis}

Representative agarose 2-DE images are shown in Figure 1A. A total of more than 2000 spots were obtained, 67 being differentially expressed between UC-associated cancer and sporadic colorectal cancer cell lines. Of the 67 spots, 2 protein spots showed a more than 2-times higher expression and 4 spots showed a more than 2-times lower expression in all $3 \mathrm{UC}$-associated cancer cell lines than in all 3 sporadic cancer cell lines. On tryptic digestion and LC-MS/MS system analysis, six proteins were identified. Heat-shock protein of $47 \mathrm{kDa}$ was detected as one protein consistently expressed higher in three UC-associated cancer cell lines than in three sporadic colorectal cancer cell lines (Figure 1B). Another protein that showed a higher expression in UC-associated cell lines was a cytoskeletal protein. Four proteins that showed a higher expression in sporadic cancer cell lines were a cytoskeletal protein, a signal transduction factor, and two enzymes associated with acetyl-coA metabolism and folate metabolism. In addition, HSP70 was detected as a protein that showed a tendency to increase in two UC cell lines, significant difference not being recognised.

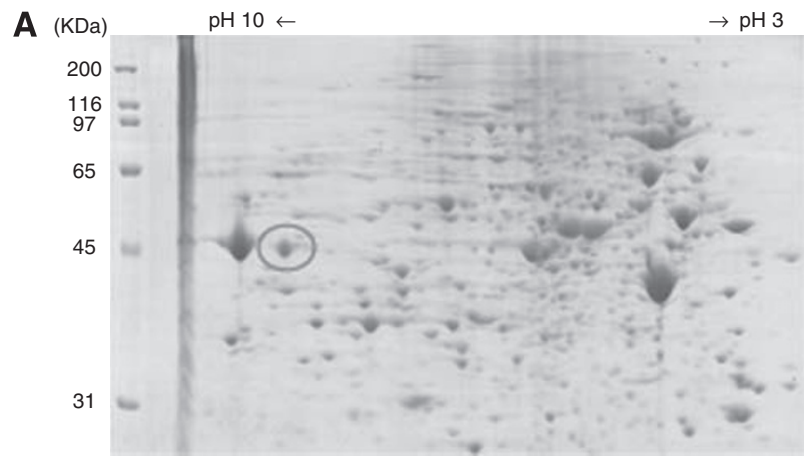

B

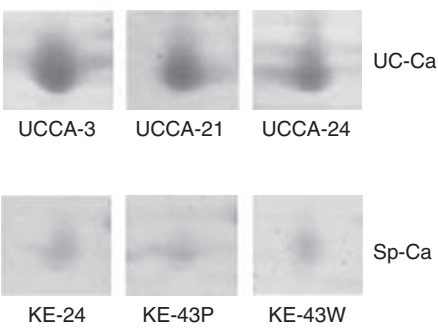

Figure I Agarose two-dimensional electrophoresis gel patterns of cell lines. (A) Representative proteome maps derived from the UCCA-3, UCassociated cancer cell line. The red circle indicates the protein spot, HSP47. (B) The zoomed two-dimensional electrophoresis gel images show expression of HSP47. UC-associated cancer cell lines show an increased expression of HSP47 compared with sporadic cancer cell lines (Coomassie blue-stained twodimensional electrophoresis gels). 
HSP47 expression by western blot analysis and immunocytochemical staining

Upregulation of HSP47 in UC-associated cancer cell lines by proteomics analysis was further confirmed using immunoblotting (Figure 2A) and immunocytochemical staining (Figure 2B). With

A

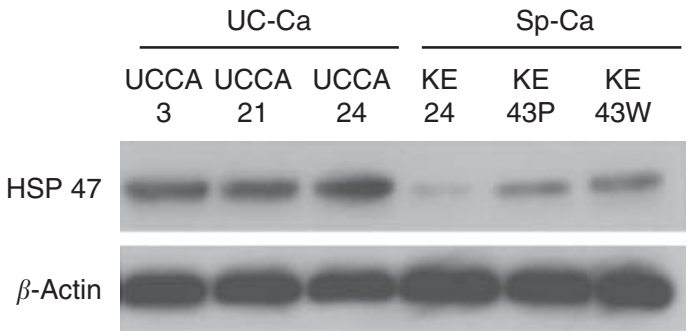

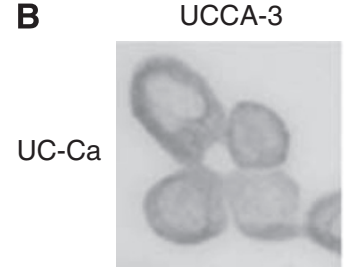

KE-24

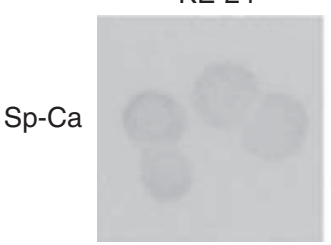

Figure 2 Western blot analysis of the HSP47 expression in cell lines (A) UC-associated cancer cell lines (UC-Ca) and sporadic cancer cell lines (Sp-Ca). $\beta$-Actin expression was used as a control. (B) Immunocytochemical staining of HSP47 in the six cell lines. The strong reactions in UC-associated cancer cell lines is noted. immunocytochemical staining, the HSP47 protein was expressed in the cytoplasm of the cells.

\section{HSP47 expression in UC-associated and sporadic colon tumours}

Typical HSP47 immunohistochemical staining patterns of UCassociated lesions are shown in Figure 3. The expression of HSP47 was lacking or very weak in normal epithelial cells. In tumour tissue, staining was evident in the cytoplasm of epithelial tumour cells. Both UC-associated and sporadic colon tumours showed a significantly higher expression than did normal mucosa. In addition, HSP47 tended to gradually increase from low-grade intramucosal lesions, dysplasia, and adenoma towards invasive carcinoma, in both series. Furthermore, UC-associated carcinoma showed a higher expression than did sporadic colonic carcinoma $(P<0.01)$, and UC-regenerative mucosa showed a significantly higher expression than did normal mucosa $(P<0.05)$ (Figure 4$)$. When UC-regenerative mucosae were histologically classified into active and inactive UC groups, there was no difference in expression between the two groups (active UC, $1.0 \pm 0.7$; inactive $\mathrm{UC}, 1.0 \pm 0.8)$.

\section{Immunofluorescent double staining of HSP47 and type I collagen}

Immunofluorescent double staining of HSP47 and type I collagen showed co-localisation in UC-associated cancer cell lines (Figure 5). In contrast, no or only a weak expression of type I collagen was found in sporadic colorectal cancer cell lines.

\section{Type I collagen synthesis measured by ELISA}

Type I collagen synthesis was shown in both UC-associated and sporadic colorectal cancer cell lines, as well as in human fibroblasts. However, compared with UC-associated cancer cell lines, collagen synthesis was at a low level in the KE 24 and KE43W cell lines (Figure 6).
A

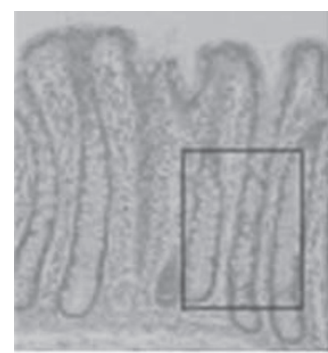

B

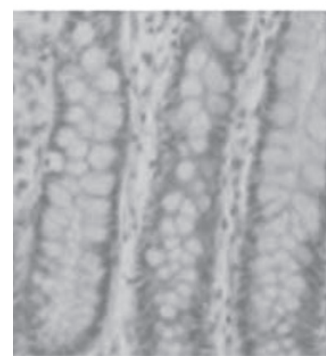

UC regenerative
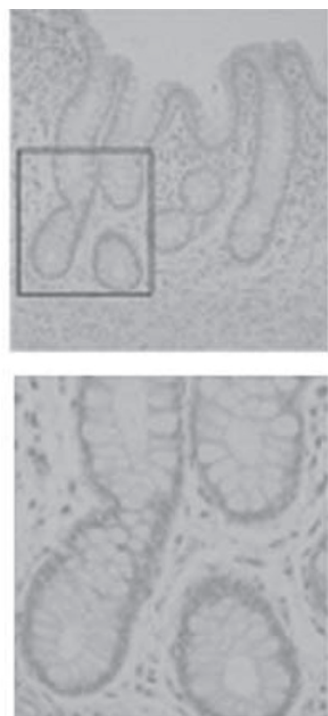

UC LGD
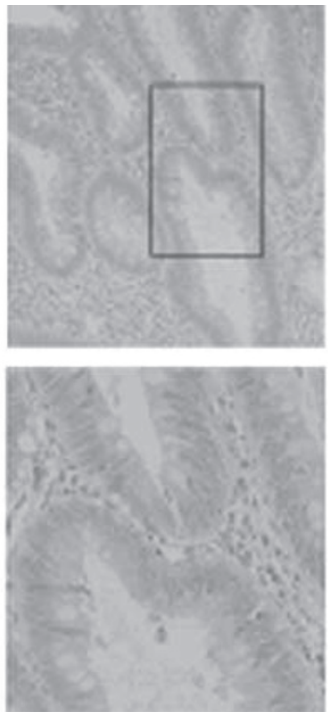

UC HGD
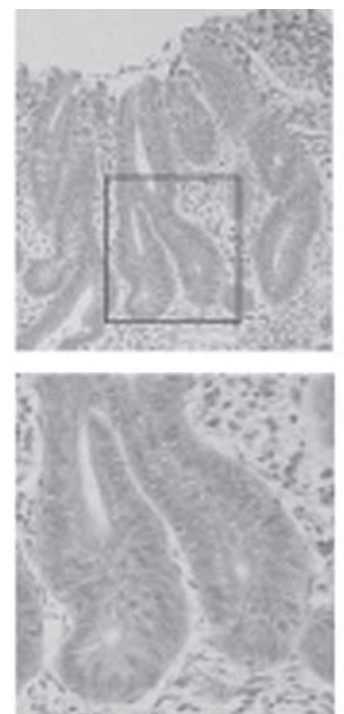

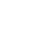




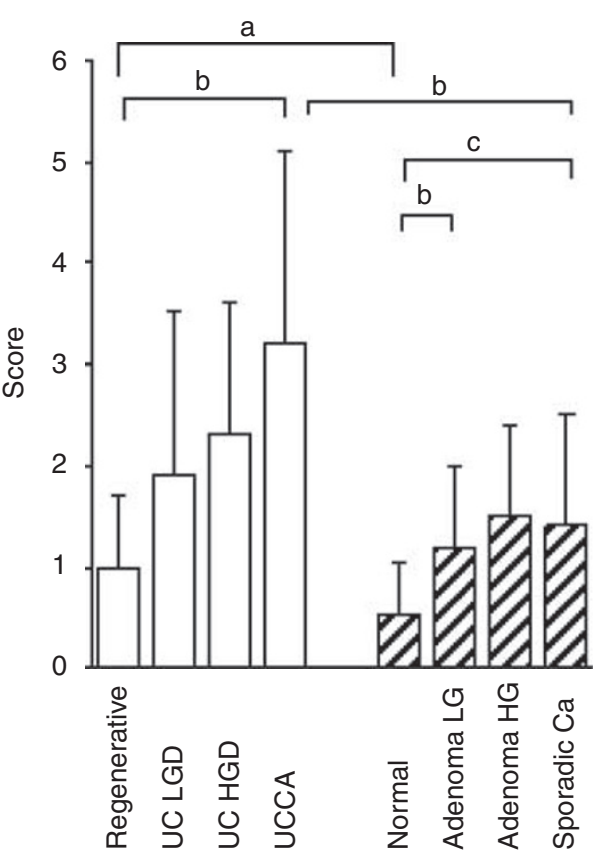

a : $P<0.05$

b : $P<0.01$

c : $P<0.001$

Figure 4 The epithelial expression of HSP47 in UC-associated (open bars) and sporadic colon tumour lesions (hatched bars). Data are mean scores and s.d. (a) $P<0.05$; (b) $P<0.0$ I; and (c) $P<0.00$ I; LGD, low-grade dysplasia; HGD, high-grade dysplasia.

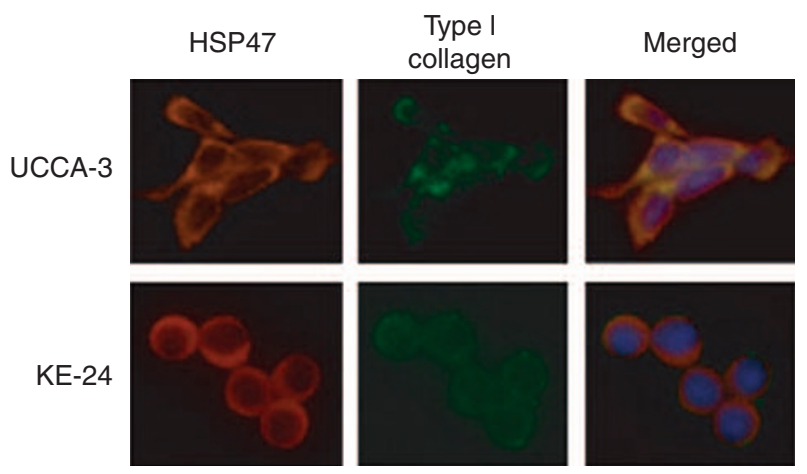

Figure 5 Double immunofluorescence staining for HSP47 (redrhodamin) and type I collagen (green-FITC) in a UC-associated cancer cell line (UCCA-3) and a sporadic cancer cell line (KE-24). The cells were counterstained with DAPI to visualise the nuclei.

\section{HSP47 in culture medium}

HSP47 was detected by immunoblotting in the culture media, particularly from UC-associated cancer cell lines. There was an extremely limited release from normal human fibroblasts (Figure 7).

\section{DISCUSSION}

Proteomic analysis of UC-associated cancer cell lines and sporadic colorectal cancer cell lines in this study showed an upregulated expression of the ER stress-response protein HSP47 in UCassociated cancer cell lines. In the culture environment, as neither inflammation nor influence of stroma exists, protein expression is limited. Therefore, we confirmed the expression using clinical materials by immunohistochemical staining. The expression of

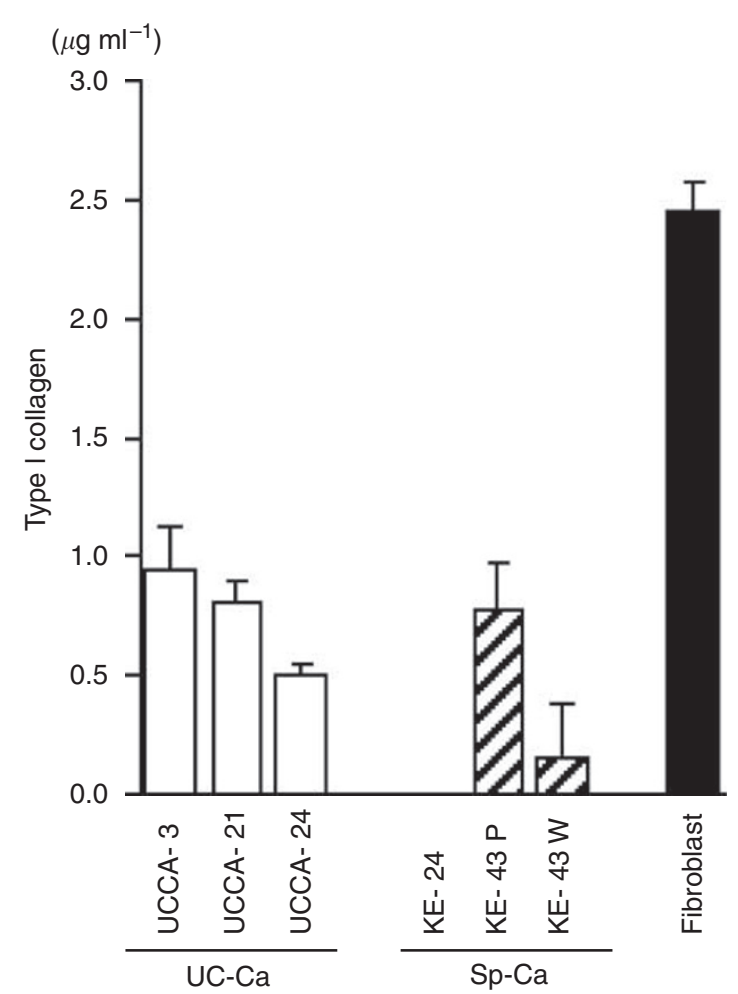

Figure 6 Collagen synthesis of UC-associated cancer cell lines (UC-Ca) (open bars), sporadic cancer cell lines (Sp-Ca) (hatched bars), and human fibroblast cells (black bars). Data are given as concentrations of type I collagen in medium. Vertical lines show s.d.

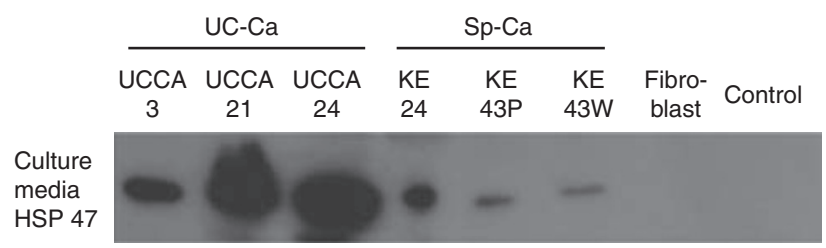

Figure 7 Western blot of HSP47 in cell culture medium. An increased release of HSP47 was shown in UC-associated cancer cell lines than in sporadic colorectal cancer cell lines.

HSP47 increased with progression through dysplasia to UCassociated carcinoma. Heat-shock protein of $47 \mathrm{kDa}$ is a collagenbinding, stress-inducible protein localised in the ER that participates in the intracellular processing, folding, assembly, and secretion of procollagens, especially in collagen-secreting cells, such as fibroblasts, for example, in fibrotic lesions (Razzaque and Taguchi, 1999). On the other hand, in epithelial or carcinoma cells, few reports have described the expression of HSP47. Our results are in line with earlier reports of overexpression of HSP47 in pancreatic carcinoma (Maitra et al, 2002), gastric carcinoma (Hirai et al, 2006), and head and neck squamous cell carcinoma (Li et al, 2008). Another chaperone protein, mortalin (mitochondrial heatshock protein 70 ), was reported as the protein that contributed to colorectal adenocarcinomas (Dundas et al, 2005). Heat-shock protein of $47 \mathrm{kDa}$ has an important role in the biosynthesis of procollagens, and co-localisation of HSP47 and collagen type I could be shown by double immunofluorescent staining in this study. In tissue, although HSP47 was found to be expressed in both epithelial and stromal cells within cancers, only the former showed an increase with progression to greater malignancy. A significantly higher expression in UC-associated than in sporadic carcinomas 
suggests that the epithelial expression of HSP47 may be influenced by background inflammation. However, as upregulation was also noted in cultured UC-associated cancer cell lines, the increase may be an intrinsic property.

In culture media, type I collagen was detected. Therefore, not only fibroblasts but also epithelial cancer cells produce type I collagen. Although the functions of collagen from epithelial cells are not clear, they may reflect stromal collagen metabolism. From the literature, inflammatory cytokine production during chronic inflammation may lead to excessive collagen deposition and eventually to fibrosis in UC (Lawrance et al, 2001a,b). Metalloproteinases may also be overexpressed and degrade stromal collagen with inflammatory bowel disease (von Lampe et al, 2000; Stumpf et al, 2006). Therefore, in chronic inflammatory condition, the biosynthesis of collagen might need to be increased chronically, with collagen produced by epithelial cells supplementing that from fibroblasts.

Heat-shock protein of $47 \mathrm{kDa}$ clearly has the potential to be a biomarker for UC-associated cancer. Although the mechanism of release from cancer cell remains to be clarified, cell death could be involved. Alternatively, a deletion of the ER retention signal or an overflow of HSP47 from the ER retention signal may cause a

\section{REFERENCES}

Dundas SR, Lawrie LC, Rooney PH, Murray GI (2005) Mortalin is overexpressed by colorectal adenocarcinomas and correlates with poor survival. J Pathol 205: 74-81

Fujii S, Katsumata D, Fujimori T (2008) Limits of diagnosis and molecular markers for early detection of ulcerative colitis-associated colorectal neoplasia. Digestion 77(Suppl 1): 2-12

Hirai K, Kikuchi S, Kurita A, Ohashi S, Adachi E, Matsuoka Y, Nagata K, Watanabe M (2006) Immunohistochemical distribution of heat shock protein 47 (HSP47) in scirrhous carcinoma of the stomach. Anticancer Res 26: 71-78

Kakugawa T, Yokota S, Mukae H, Kubota H, Sakamoto N, Mizunoe S, Matsuoka Y, Kadota J, Fujii N, Nagata K, Kohno S (2008) High serum concentrations of autoantibodies to HSP47 in nonspecific interstitial pneumonia compared with idiopathic pulmonary fibrosis. BMC Pulm Med 8: 23

Kuruma H, Egawa S, Oh-Ishi M, Kodera Y, Maeda T (2005) Proteome analysis of prostate cancer. Prostate Cancer Prostatic Dis 8: 14-21

Lawrance IC, Maxwell L, Doe W (2001a) Altered response of intestinal mucosal fibroblasts to profibrogenic cytokines in inflammatory bowel disease. Inflamm Bowel Dis 7: 226-236

Lawrance IC, Maxwell L, Doe W (2001b) Inflammation location, but not type, determines the increase in TGF-betal and IGF-1 expression and collagen deposition in IBD intestine. Inflamm Bowel Dis 7: 16-26

Lawrie LC, Fothergill JE, Murray GI (2001) Spot the differences: proteomics in cancer research. Lancet Oncol 2: 270-277

Li D, Guang W, Abuzeid WM, Roy S, Gao GP, Sauk JJ, O'Malley Jr BW (2008) Novel adenoviral gene delivery system targeted against head and neck cancer. Laryngoscope 118: 650-658

Maitra A, Iacobuzio-Donahue C, Rahman A, Sohn TA, Argani P, Meyer R, Yeo CJ, Cameron JL, Goggins M, Kern SE, Ashfaq R, Hruban RH, Wilentz RE (2002) Immunohistochemical validation of a novel epithelial and a novel stromal marker of pancreatic ductal adenocarcinoma identified by global expression microarrays: sea urchin fascin homolog and heat shock protein 47. Am J Clin Pathol 118: 52 - 59

Nagata K, Saga S, Yamada KM (1986) A major collagen-binding protein of chick embryo fibroblasts is a novel heat shock protein. J Cell Biol 103: $223-229$

Nakai A, Satoh M, Hirayoshi K, Nagata K (1992) Involvement of the stress protein HSP47 in procollagen processing in the endoplasmic reticulum. J Cell Biol 117: $903-914$ leakage of HSP47 to outside the cell (Yokota et al, 2003). Only very limited release was noted from cultured fibroblasts. Recently, highserum auto-antibodies to HSP47 or HSP47 itself have been reported under inflammatory conditions, such as interstitial pneumonia and mixed connective tissue disease (Yokota et al, 2003; Kakugawa et al, 2008). If HSP47 is released from cancers in patients with UC, its detection in serum would be helpful for the clinical detection of malignancy. As surveillance by endoscopic examination sometimes fails to detect cancer lesions (Fujii et al, 2008), an easily measurable biomarker would clearly be very welcome.

In conclusion, overexpression of HSP47 is a unique characteristic of UC-associated carcinoma. Further studies are required to elucidate its clinical significance.

\section{ACKNOWLEDGEMENTS}

We thank Dr Yoshimitsu Kuroyanagi and Kazuya Yamashita for the gifts of cells used in this study. We are also grateful to $\mathrm{Dr}$ Malcolm Moore for careful reading and English editing of this manuscript.
Oh-Ishi M, Satoh M, Maeda T (2000) Preparative two-dimensional gel electrophoresis with agarose gels in the first dimension for high molecular mass proteins. Electrophoresis 21: 1653-1669

Okayasu I, Hana K, Yoshida T, Mikami T, Kanno J, Fujiwara M (2002) Significant increase of colonic mutated crypts in ulcerative colitis correlatively with duration of illness. Cancer Res 62: 2236-2238

Razzaque MS, Taguchi T (1999) The possible role of colligin/HSP47, a collagen-binding protein, in the pathogenesis of human and experimental fibrotic diseases. Histol Histopathol 14: 1199- 1212

Sada M, Igarashi M, Yoshizawa S, Kobayashi K, Katsumata T, Saigenji K, Otani Y, Okayasu I, Mitomi H (2004) Dye spraying and magnifying endoscopy for dysplasia and cancer surveillance in ulcerative colitis. Dis Colon Rectum 47: 1816-1823

Sobin LH, Wittekind C (2002) UICC TNM Classification of Malignant Tumours, 6th edn: New York: Wiley-Liss

Stumpf M, Krones CJ, Klinge U, Rosch R, Junge K, Schumpelick V (2006) Collagen in colon disease. Hernia 10: 498-501

Tokuyama W, Mikami T, Fujiwara M, Matsui T, Okayasu I (2007) Midkine expression in colorectal tumors: correlation with Ki-67 labeling in sporadic, but not ulcerative colitis-associated ones. Pathol Int 57: 260-267

Tomonaga T, Matsushita K, Yamaguchi S, Oh-Ishi M, Kodera Y, Maeda T, Shimada H, Ochiai T, Nomura F (2004) Identification of altered protein expression and post-translational modifications in primary colorectal cancer by using agarose two-dimensional gel electrophoresis. Clin Cancer Res 10: 2007-2014

von Lampe B, Barthel B, Coupland SE, Riecken EO, Rosewicz S (2000) Differential expression of matrix metalloproteinases and their tissue inhibitors in colon mucosa of patients with inflammatory bowel disease. Gut 47: 63-73

Wong NA, Harrison DJ (2001) Colorectal neoplasia in ulcerative colitisrecent advances. Histopathology 39: 221-234

Yamashita K, Yasuda S, Kuba T, Otani Y, Fujiwara M, Okayasu I (2004) Unique characteristics of rectal carcinoma cell lines derived from invasive carcinomas in ulcerative colitis patients. Cancer Sci 95: $211-217$

Yokota S, Kubota H, Matsuoka Y, Naitoh M, Hirata D, Minota S, Takahashi H, Fujii N, Nagata K (2003) Prevalence of HSP47 antigen and autoantibodies to HSP47 in the sera of patients with mixed connective tissue disease. Biochem Biophys Res Commun 303: $413-418$ 